\title{
Gastric Diverticula: A Review and Report of Two Cases
}

\author{
Daryl Ramai ${ }^{\mathrm{a}, \mathrm{b}}$, Andrew Ofosu ${ }^{\mathrm{a}}$, Madhavi Reddy ${ }^{\mathrm{a}}$
}

\begin{abstract}
Gastric diverticula represent the rarest of gastrointestinal diverticula. While similar to duodenal, jejunal and colonic diverticula, gastric diverticula are found incidentally upon upper endoscopy and are mostly asymptomatic. However, larger diverticula can lead to symptoms such as upper abdominal pain, nausea, emesis, and dyspepsia. Some patients may present with dramatic presentations including ulceration, hemorrhage, or perforation. Clinicians should be aware that diagnostic imaging may not always detect the presence of gastric diverticula. Upper endoscopy is recommended for diagnosing gastric diverticula as it readily confirms the size and location of the lesion. Conservative treatment involves the use of antacids and may provide temporary relief. Surgical resection is recommended for treatment resistant gastric diverticula, or cases complicated by hemorrhage, perforation, or malignancy. Herein, we present two patients with antral gastric diverticula, and review the literature.
\end{abstract}

Keywords: Gastric diverticulum; Endoscopy; Abdominal pain; Dysphagia; Gastric; Diverticulum

\section{Introduction}

Gastric diverticulum is characterized as a pouch within the gastric wall, similar to duodenal, jejunal and colonic diverticula. Gastric diverticula represent the rarest form of gastrointestinal diverticula, with an estimated prevalence of $0.04 \%$ in contrast radiographs and $0.02 \%$ using autopsy studies $[1$, $2]$. The discrepancy in prevalence reflects the use of different diagnostic modalities. However, gastric diverticula are distributed equally among male and female patients, and approximately $82 \%$ of cases occur over 40 years of age [3]. In addition, approximately $4 \%$ of gastric diverticula occur in pa-

Manuscript submitted November 13, 2017, accepted December 6, 2017

aDivision of Gastroenterology and Hepatology, The Brooklyn Hospital Center, Academic Affiliate of The Icahn School of Medicine at Mount Sinai, Clinical Affiliate of The Mount Sinai Hospital, 121 Dekalb Avenue, Brooklyn, NY 11201, USA

${ }^{\mathrm{b} C}$ Corresponding Author: Daryl Ramai, Division of Gastroenterology and Hepatology, The Brooklyn Hospital Center, Academic Affiliate of The Icahn School of Medicine at Mount Sinai, Clinical Affiliate of The Mount Sinai Hospital, 121 Dekalb Avenue, Brooklyn, NY 11201, USA. Email: dramai@tbh.org

doi: https://doi.org/10.14740/gr936w tients younger than 20 years old, with cases reported in newborns $[2,4]$. Herein, we report two patients with antral gastric diverticula.

\section{Case Reports}

\section{Case 1}

A 44-year-old male with a past medical history of hypertension and asthma was admitted for abdominal pain and hematochezia. The patient reported having abdominal pain after eating and drinking. He described having diffuse abdominal pain and persistent bloody stools for 3 weeks, and denied any associated fever, nausea, chills, or weight loss. The patient also denied smoking, alcohol and illicit drug use. Abdominal examination was soft, non-tender, and non-distended. Laboratory findings showed white blood cell count was $9.4 \times 10^{3} / \mu \mathrm{L}$ (normal range: $\left.4.8-10.8 \times 10^{3} / \mu \mathrm{L}\right)$, hemoglobin $13.3 \mathrm{~g} / \mathrm{dL}(12.0-16.0$ $\mathrm{g} / \mathrm{dL})$, hematocrit $41 \%(37-47 \%)$, and platelet count $240 \times 10^{3} /$ $\mu \mathrm{L}\left(130-400 \times 10^{3} / \mu \mathrm{L}\right)$. Blood urea nitrogen and creatinine were normal at $11 \mathrm{mg} / \mathrm{dL}(8-27 \mathrm{mg} / \mathrm{dL})$ and $1.2 \mathrm{mg} / \mathrm{dL}(0.8$ - $2.0 \mathrm{mg} / \mathrm{dL}$ ), respectively. Vital signs were stable and within normal limits.

Abdominal computer tomography (CT) scan was suspicious for possible pan-colitis and inflamed lymph nodes. Diagnostic colonoscopy showed inflammation in the cecum which was also suggestive of colitis, while the colon appeared normal. Upper endoscopy showed mild erythematous mucosa of the stomach. Biopsy specimens revealed mild gastritis without evidence of Helicobacter pylori. A diverticulum was found in the antrum of the stomach, which appeared to have normal appearing mucosa (Fig. 1). An upper gastrointestinal series (UGS) to further characterize its morphology was not performed. However, UGS would be recommended if the patient elected to have surgical resection of the diverticulum.

\section{Case 2}

A 32-year-old female with a past medical history of dyspepsia presented to the outpatient gastroenterology clinic with complaints of bloating and abdominal gas despite proton pump inhibitor (PPI) therapy. The patient also reported experiencing early satiety, no significant reflux, and occasional vomiting. She denied any associated fever, nausea, chills, or weight loss. The patient also denied smoking, but reported occasional binge drinking. Abdominal examination was soft, non-tender, and 


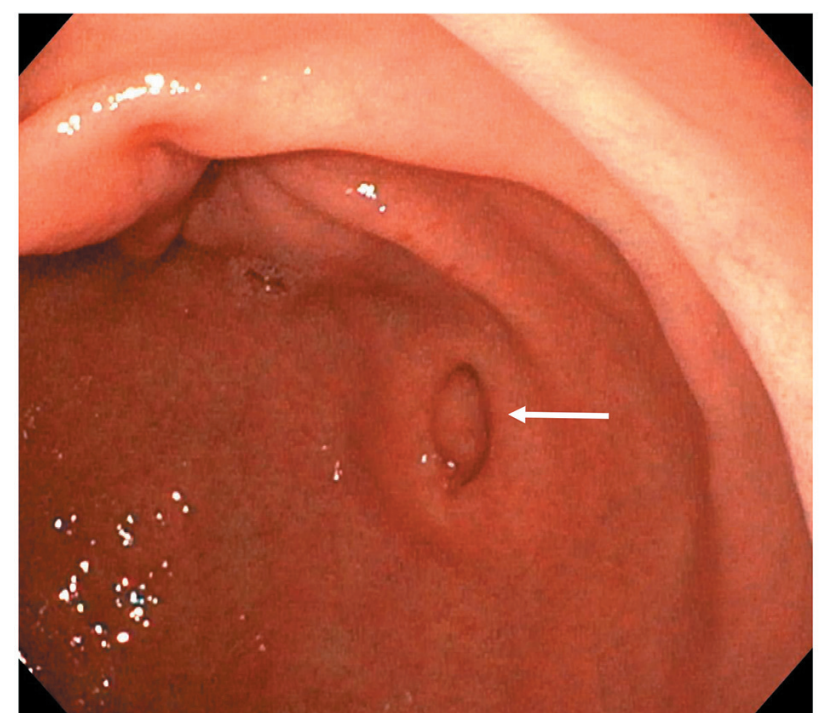

Figure 1. Endoscopic view of gastric diverticulum (white arrow) located in the antrum.

non-distended. Laboratory findings showed white blood cell count was $3,700 / \mathrm{mm}^{3}$ (normal range: $4,800-10,800 / \mathrm{mm}^{3}$ ), hemoglobin $12.1 \mathrm{~g} / \mathrm{dL}(12.0$ - $16.0 \mathrm{~g} / \mathrm{dL})$, hematocrit $37 \%$ (37$47 \%)$, and platelet count $188,000 / \mathrm{mm}^{3}(130,000-400,000 /$ $\mathrm{mm}^{3}$ ). Blood urea nitrogen and creatinine were normal at 7 $\mathrm{mg} / \mathrm{dL}(8-27 \mathrm{mg} / \mathrm{dL})$ and $0.7 \mathrm{mg} / \mathrm{dL}(0.8-2.0 \mathrm{mg} / \mathrm{dL})$, respectively. Vital signs were stable and within normal limits.

Upper endoscopy showed mild erythematous mucosa of the stomach. Biopsy specimens revealed mild gastritis without evidence of Helicobacter pylori. A gastric diverticulum was found in the antrum, which appeared to have normal appearing mucosa. Upon further entry, there appeared to be two smaller diverticula within the larger diverticulum (Fig. 2). In this case, a UGS was performed to characterize the morphology of the diverticulum which returned normal.

\section{Discussion}

Gastric diverticula were first described by Moebius in 1661 and later by Roax in 1774 [5]. They are usually singular lesions and range in size from 1 to $5 \mathrm{~cm}$ in diameter with occasional reports describing lesions of $10-11 \mathrm{~cm} \mathrm{[6].} \mathrm{Ap-}$ proximately $74-90 \%$ of diverticula occur in the gastric cardia, while antral diverticula are rare [3]. Gastric diverticula can be grouped into two types, namely, congenital (true) and acquired (false).

Congenital diverticula are more common, and involve all layers of the gastric wall. This arises from the splitting of longitudinal muscular fibers, leaving only circular muscle fibers in the gastric wall. As a result, a weakening occurs within the wall of the stomach and allows a diverticulum to form, particularly during the fetal period [6]. In contrast, false diverticula do not affect all layers of the gastric wall, and are classified as pulsation and traction diverticula. Pulsation diverticula result from intraluminal pressure due to chronic coughing, obesity

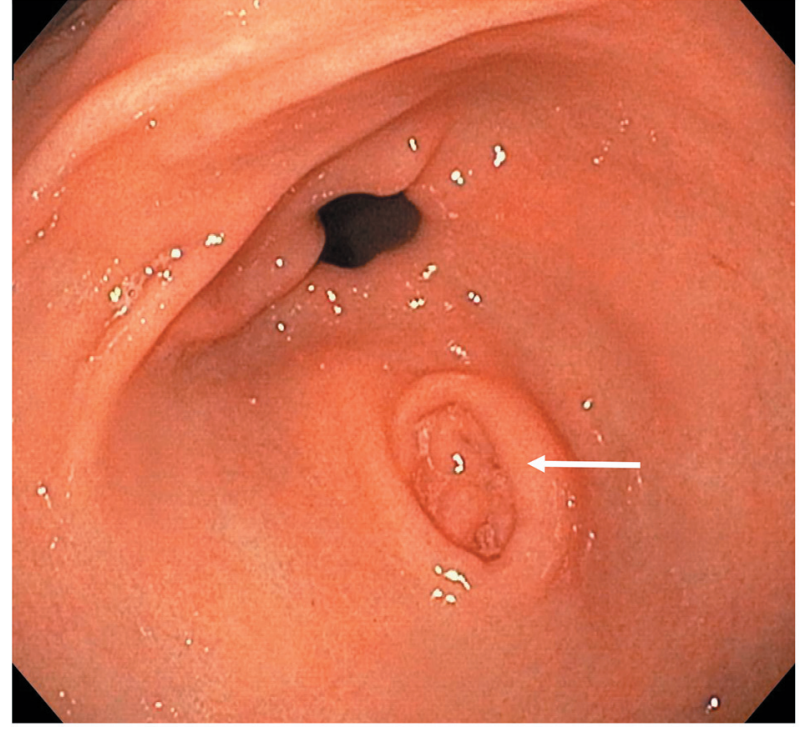

Figure 2. Endoscopic view of gastric diverticulum (white arrow) located in the antrum. Two smaller diverticula can be seen within the larger diverticulum.

and pregnancy. Traction diverticula arise from perigastric adhesions from concurrent diseases, such as peptic ulcer disease, pancreatitis, malignancy, gastroesophageal reflux, and cholecystitis. Most congenital diverticula $(70 \%)$ are located on the posterior wall of the stomach just below the gastroesophageal junction, while acquired diverticula are typically near the gastric antrum [7].

Most cases of gastric diverticula are asymptomatic, probably because of the posterior location and broad base. However, depending on the size of the diverticula, patients may present with non-specific symptoms such as abdominal pain, emesis, weight loss, iron deficiency anemia, or complications such as bleeding, gastroesophageal reflux, and perforation [6, 7]. Dyspepsia and vomiting are less commonly reported. Furthermore, the diagnosis of gastric diverticula can be challenging as it can be missed on imaging studies. UGS can be used to characterize the extent of the diverticula and the presence of a mucosal sac. However, lesions can be missed with UGS, as reported in case 2. Upper endoscopy is recommended for diagnosing gastric diverticula as it readily confirms the size and location of the lesion.

Management of gastric diverticula is dependent on the presence of symptoms. Incidental findings of gastric diverticula or asymptomatic cases require no specific treatment. Conservative therapy with antacids provides temporary symptomatic relief and may not resolve the underlying pathology [8]. Lesions greater than $4 \mathrm{~cm}$ are more likely to produce complications, are less responsive to medications, and may require surgical intervention. In 30 of 49 symptomatic patients with gastric diverticula, symptoms were attributable to other gastrointestinal diseases, and six of nine patients with symptoms caused by a gastric diverticulum who underwent open surgery showed excellent outcomes [2]. While several surgical approaches have been described in the literature, laparoscopic resection is the preferred method of choice $[9,10]$. To this end, 
simple resection of the diverticulum with a laparoscopic cutting stapler has been successful.

\section{Conclusion}

Gastric diverticulum is a rare endoscopic finding. We report two patients, case 1 without symptoms, and case 2 with symptoms of dysphagia. While dysphagia can have multiple etiologies, it can be a symptom of a gastric diverticulum. Both patients were treated with anti-reflux medications and followed up in the outpatient clinic. Clinicians should have a high clinical index of suspicion to diagnose patients with gastric diverticulum. This condition typically presents with a long history of vague symptoms such as upper abdominal pain and dyspepsia. It does not always resolve with PPIs and can even be missed on upper endoscopy, CT scanning, or UGS. Symptomatic patients, non-responsive to medical therapy, are recommended laparoscopic repair as a safe and effective procedure, not responded to medical therapy.

\section{Conflict of Interest}

None.

\section{Funding}

None.

\section{Consent}

Obtained.

\section{Author Contributions}

$\mathrm{AO}$ and DR conceived and designed the paper. DR drafted the manuscript. AO and MR edited the manuscript for intellectual content.

\section{References}

1. Rivers A, Stevens G, Kirklin B. Diverticula of the stomach. Surg Gynecol Obstet. 1935;60:106-113.

2. Palmer ED. Gastric diverticula. Int Abstr Surg. 1951;92(5):417-428.

3. Brown CH, Bissonnette RP, Albee RD. Diverticula of the stomach; a report of 30 cases and a review of the literature. Gastroenterology. 1949;12(1):10-23.

4. Rashid F, Aber A, Iftikhar SY. A review on gastric diverticulum. World J Emerg Surg. 2012;7(1):1.

5. Moses WR. Diverticula of the stomach. Arch Surg. 1946;52:59-65.

6. Lewis FT, Thyng FW. Regular occurrence of intestinal diverticula in embryos of pig, rabbit and man. Am J Anat. 1908; 7:505.

7. Rodeberg DA, Zaheer S, Moir CR, Ishitani MB. Gastric diverticulum: a series of four pediatric patients. J Pediatr Gastroenterol Nutr. 2002;34(5):564-567.

8. Marano L, Reda G, Porfidia R, Grassia M, Petrillo M, Esposito G, Torelli F, et al. Large symptomatic gastric diverticula: two case reports and a brief review of literature. World J Gastroenterol. 2013;19(36):6114-6117.

9. DuBois B, Powell B, Voeller G. Gastric diverticulum: "a wayside house of ill fame" with a laparoscopic solution. JSLS. 2012;16(3):473-477.

10. Rashid F, Singh R, Cole A, Iftikhar SY. Troublesome belching with fetor odour. Gut. 2010;59(3):310, 324. 